\title{
TEXTURE AND MICROSTRUCTURE OF COMPRESSED COPPER
}

\author{
C. S. LEE, C. F. LEE and R. E. SMALLMAN* \\ Department of Physics and Materials Science, \\ City University of Hong Kong, 83 Tat Chee Avenue, Hong Kong \\ *School of Metallurgy and Materials, University of Birmingham, \\ Birmingham, B15 2TT, UK
}

(Received 16 November 1995)

\begin{abstract}
It is shown that the deformation banding tendency in copper increases as deformation temperature increases. After 70\% compression, the average number of deformation bands per grain increases from 16 to 36 as the deformation temperature increases from 25 to $300^{\circ} \mathrm{C}$. An orientation dependence of banding tendency is also observed. Grains with near $\{110\}$ orientation are found to have the lowest banding tendency. The textures of samples compressed below $300^{\circ} \mathrm{C}$ are similar and consist mainly of a $\{110\}$ fibre and a weak $\{100\}$ fibre. While the $\{110\}$ component remains when deformation temperature is increased to $300^{\circ} \mathrm{C}$; the $\{100\}$ component becomes weaker. $300^{\circ} \mathrm{C}$ is in fact the temperature at which large scale dynamic recrystallisation occurs. It is suspected that the decay of the $\{100\}$ component is due to dynamic recrystallisation which takes place preferentially at thin deformation bands where most of the $\{100\}$ oriented material is found.
\end{abstract}

KEY WORDS: Compression, texture, deformation banding, temperature effect.

\section{INTRODUCTION}

In the history of texture research, Prof. $\mathrm{H}$. $\mathrm{Hu}$ is no doubt an important key figure. His contribution can easily be found in most of the important topics of the field. These include recognising the importance of stacking fault frequency in deformation texture transition (Hu and Cline, 1961; Hu, Cline and Goodman, 1961; Hu and Goodman, 1963; Goodman and $\mathrm{Hu}, 1964)$, studies on deformation banding formation ( $\mathrm{Hu}, 1961,1963$; $\mathrm{Hu}$ and Cline, 1965), observation of cube grain nucleation in transition bands (Hu, 1963) and pointing out the importance of using TEM foils perpendicular to the rolling plane (Hu, 1969).

The main subject of the present studies is the formation of deformation bands, the importance of which has long be recognised by Prof. $\mathrm{Hu}(\mathrm{Hu}, 1961,1963 ; \mathrm{Hu}$ and Cline, 1965) and other researchers including Barrett et al. $(1939,1940,1943)$ and Chin (1969). While interest in the topic has been relatively low in the past two decades, the formation and influence of deformation banding and other grain subdivision processes have significant attention recently (Bay, Hansen and Kuhlmann-Wilsdorf, 1989; Hughes and Hansen, 1991; Akef and Driver, 1991; Bay et al., 1992; Maurice and Driver, 1993; Lee and Duggan, 1993; Lee, Duggan and Smallman, 1993a, b; Rosen et al., 1995). Lee, Duggan and Smallman (1993a) pointed out that the energy required to form a new deformation band boundary can be provided by a saving in plastic work due to a reduction in the number of slip systems activated. Mott (1951) has, however, 
pointed out that dislocations which build up deformation band boundaries are usually not in a low energy configuration. As the deformation temperature increases, it should be possible for these boundary dislocations to rearrange themselves into a lower energy configuration. Hence, the boundary energy should decrease as deformation temperature increases. This in turn suggests that the formation of deformation bands is easier as the temperature increases (Lee, Duggan and Smallman, 1993a).

The purpose of the present work is to determine systematically how texture, microstructure and, in particular, the formation of deformation bands are influenced by deformation temperature.

\section{EXPERIMENTAL}

Hot rolled high purity copper with an average grain size of $300 \mu \mathrm{m}$ and a weak initial texture was cut into rectangular samples of dimensions $19 \times 10 \times 10 \mathrm{~mm}$ with a high speed diamond saw. Deformed surface layers due to cutting were removed by immersing the samples into a bath of $50 \%$ nitric acid for one minute. The samples were then compressed with a hydraulic press at various temperatures (room, $150,200,250,300^{\circ} \mathrm{C}$ ) to about $70 \%$ thickness reduction at a strain rate of about $0.02 \mathrm{~s}^{-1}$. Molykote (produced by Dow Corning) was applied to both ends of the compression samples to reduce end friction. All samples were quenched into water within five seconds after compression.

Microstructural studies were carried out on transverse sections of the compressed samples. To minimise the influence of end friction during compression, only the central region of each sample was used for any observation or measurement. Concentrated nitric acid, which attacks copper crystallographically (Köhlhoff, Sun and Lücke, 1988) was used as etchant to reveal the microstructure as well as any orientation information of the compressed samples.

It was found during the studies that deformation banding is a characteristic structure in the compressed samples. While in some cases, deformation bands can be distinguish easily from those simply compressed grains, there is difficulty in other cases to judge whether a layer of consistent uniform orientation is a compressed grain or a thick deformation band with sharp boundary. These grains or bands of uniform orientations are generally referred as orientation layers in this paper.

To assess the banding tendency at various deformation temperatures, average thicknesses of orientation layers were measured. The measurement for each deformation temperature was obtained from at least two samples scanned over a total distance of about 100 times the corresponding average layer thickness (i.e. the sampling length varies from several millimetres to $730 \mu \mathrm{m}$ for the samples deformed at room temperature to $300^{\circ} \mathrm{C}$ ).

The orientation of the individual orientation layer, from the same sampling regions as the average thickness measurement, was determined from the respective etching pattern and plotted on inverse pole figures. However, it should be emphasised that texture data presented here is only considered to be a qualitative rather than quantitative representation of the true textures for two reasons. Firstly, the accuracy of the etching technique (about 2.5 to $10^{\circ}$, depending on the orientation) is lower than that of the $\mathrm{X}$-ray or electron diffraction methods. The second reason is that each orientation layer is represented by a point in an inverse pole figure regardless of its layer thickness. Hence, the inverse pole figures simply show the count of orientations rather than their volume fractions. While more quantitative measurements are underway (Lee et al., to 
be published), the present results are already useful for deducing certain information on the deformation behaviour of the material.

\section{RESULTS AND DISCUSSIONS}

\section{Microstructure}

Figure 1a shows the general microstructure of a sample compressed $70 \%$ at room temperature. The most interesting feature is that the thicknesses of orientation layers are considerably smaller than the average grain thickness expected $(300 \times 0.3=90$ $\mu \mathrm{m})$ as a result of homogeneous deformation. In fact, regions of a single orientation thicker than $50 \mu \mathrm{m}$ were seldom observed. Instead, most of the orientation layers are of only 5 to $40 \mu \mathrm{m}$ thick. This reduction in thickness scale is due to subdivision of grains into deformation bands which can be observed in most grains (Figure 1b). The microstructures of samples compressed at 150 and $200^{\circ} \mathrm{C}$ show no qualitative difference from those of the samples deformed at room temperature.

As the deformation temperature increases to $250^{\circ} \mathrm{C}$, two different types of microstructures were observed. In some regions (Figure 2a), the microstructure is similar to those found in samples compressed at lower temperatures. The only difference being that orientation layers are thinner in samples compressed at $250^{\circ} \mathrm{C}$. However, in other regions (Figure 2b), the microstructure is more turbulent and it appears that original grains have broken into tiny fragments. Isolated dynamically recrystallised grains were occasionally observed in both types of microstructures. When the deformation temperature increases to $300^{\circ} \mathrm{C}$, the microstructure consists of thin orientation layers embedded with clusters of dynamically recrystallised grains (Figure 3).

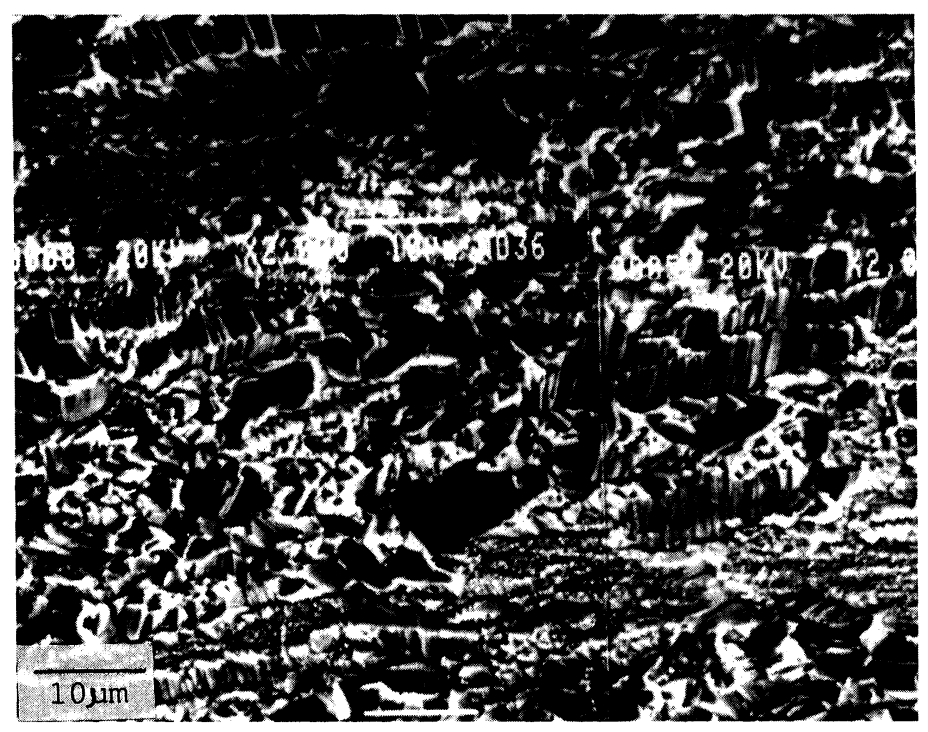

Figure 3 Transverse section of medium-grain-sized copper compressed $70 \%$ at $300^{\circ} \mathrm{C}$ showing dynamically recrystallised grain clusters surrounded by layered deformation structure. 

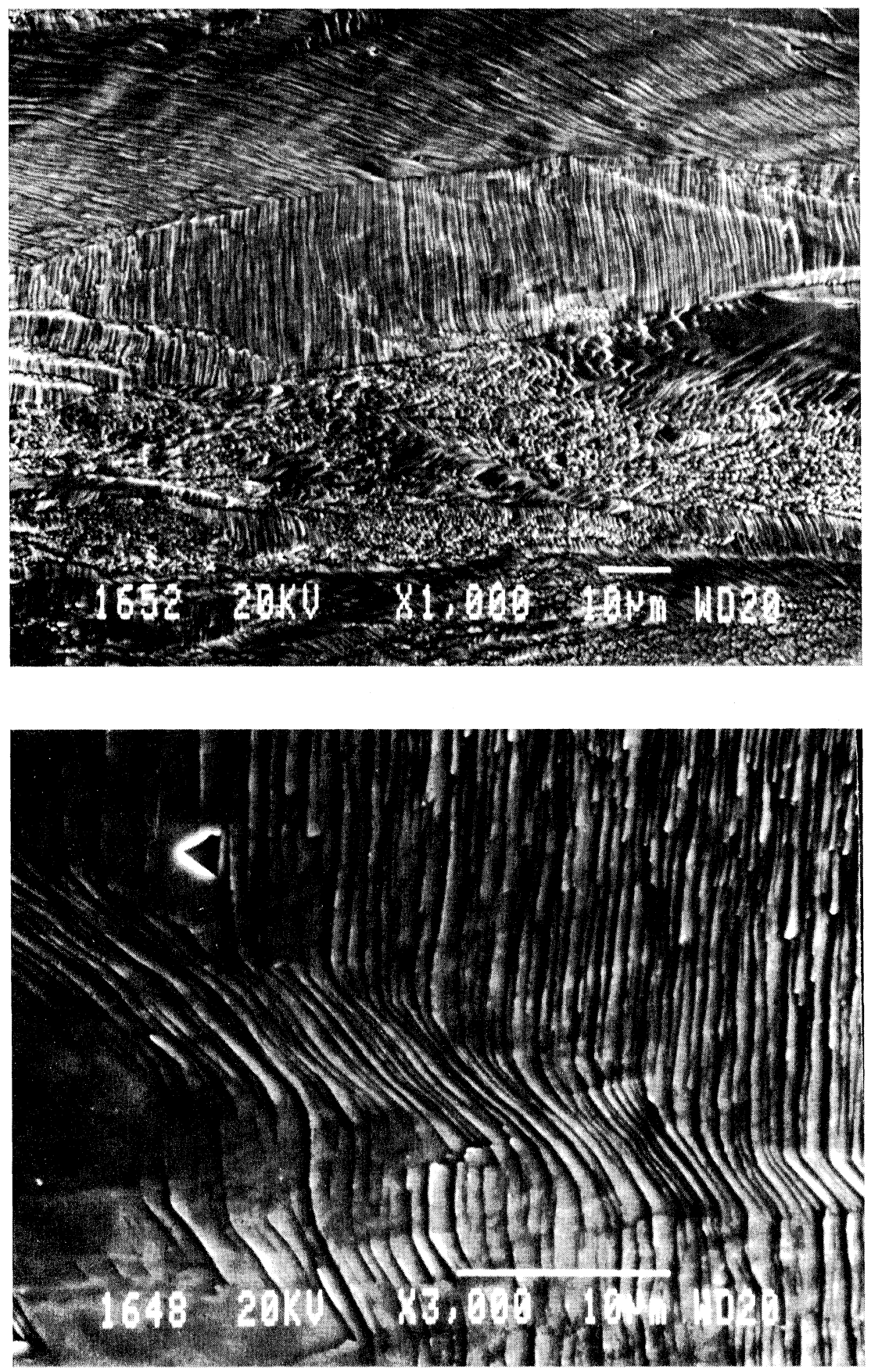

Figure 1 Transverse section of medium-grain-sized copper compressed $70 \%$ at room temperature showing a) general microstructure and b) a grain subdivided into deformation bands of different orientations. 

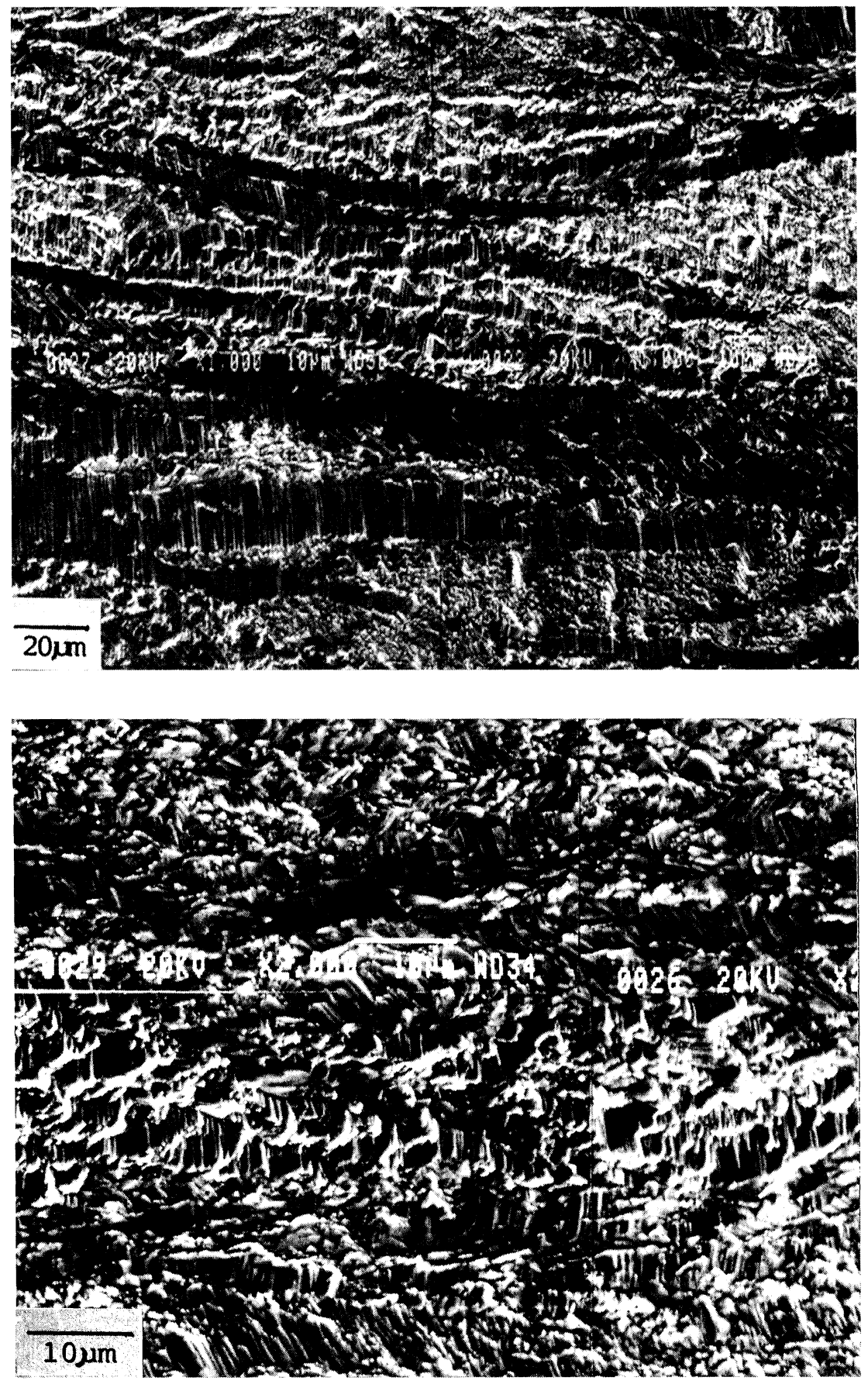

Figure 2 Transverse section of medium-grain-sized copper compressed $70 \%$ at $250^{\circ} \mathrm{C}$ showing a) a regular layer structure and b) a more turbulent structure of grain fragments. 

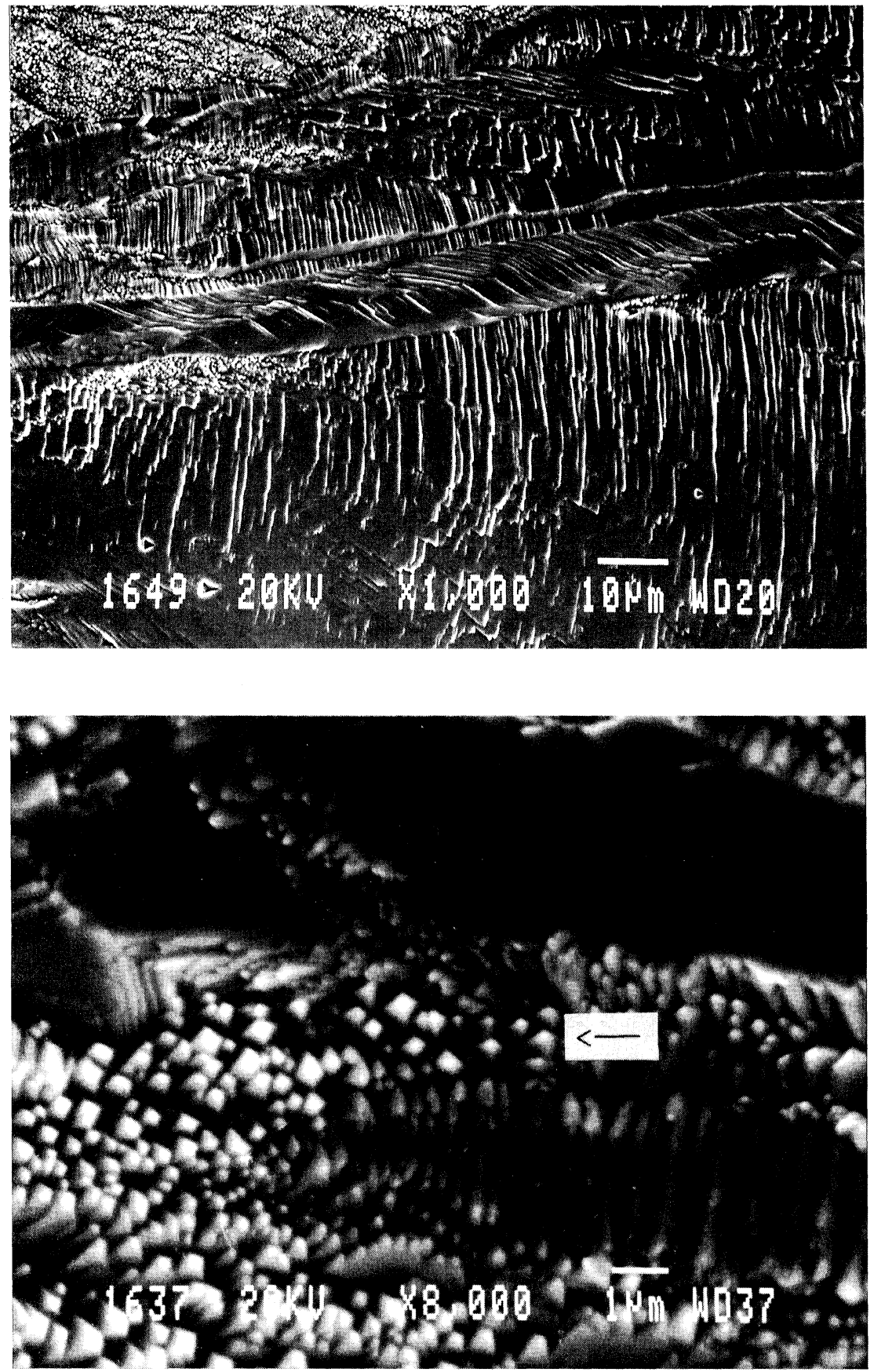

Figure 4 Transverse section of medium-grain-sized copper compressed $70 \%$ at room temperature showing a) a thick $\{110\}$ oriented grain and b) a thin $\{100\}$ oriented deformation band (region with the pyramid etching pattern marked by an arrow). 
A common observation for samples deformed at all temperatures is that orientation layers with near $\{110\}$ orientations (Figure 4a) are usually thicker than those with other orientations. On the other hand, layers with near $\{100\}$ orientations usually exist as thin and tiny fragments (Figure 4b).

To summarise, the microstructure after $70 \%$ compression consists mainly of flat grains subdivided by deformation banding with the subdivision scale decreases as deformation temperature increases up to $300^{\circ} \mathrm{C}$ at which temperature dynamic recrystallisation occurs.

\section{Thickness of orientation layers and deformation banding frequency}

The above general microstructural observation suggests that grains are subdivided to give more deformation bands as deformation temperature increases. This increasing banding frequency can be verified quantitatively by plotting the average orientation layer thickness against deformation temperature as shown in Figure 5. These average thickness values imply that each grain is deformation banded to give an average of $16,20,21$, 25 and 36 orientation layers when the deformation temperature is $25,150,200,250$ and $300^{\circ} \mathrm{C}$ respectively.

Figure 6 shows the volume fractions of orientation layers with various thicknesses. It can be observed that in samples deformed at room temperature, most orientation layers have thicknesses between 10 to $20 \mu \mathrm{m}$. For the 150 and $200^{\circ} \mathrm{C}$ deformed samples, most layers have thicknesses between 5 to $10 \mu \mathrm{m}$. Most of the orientation layer are thinner than $5 \mu \mathrm{m}$ in samples compressed at 250 and $300^{\circ} \mathrm{C}$.

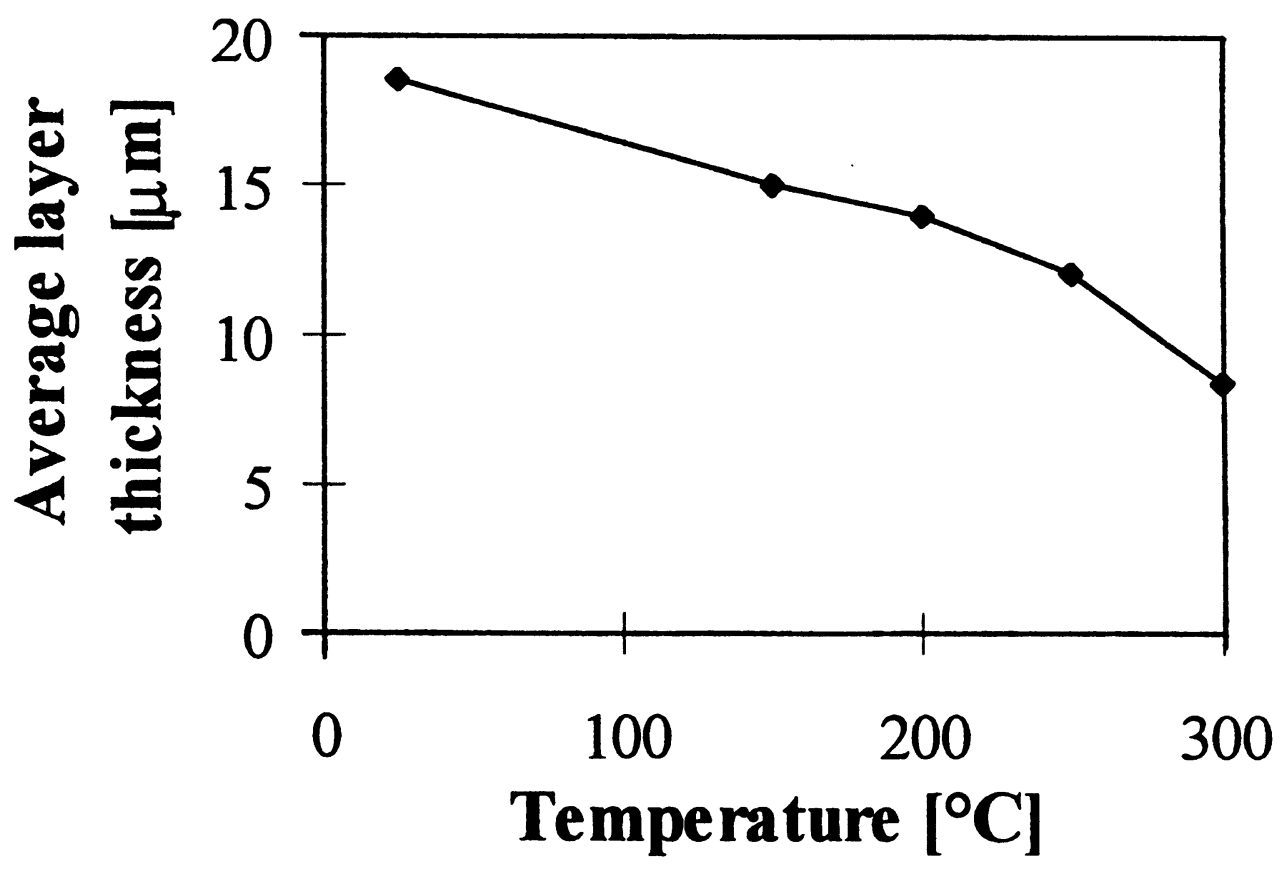

Figure 5 A graph of average orientation layer thickness after $70 \%$ compression against deformation temperature. 


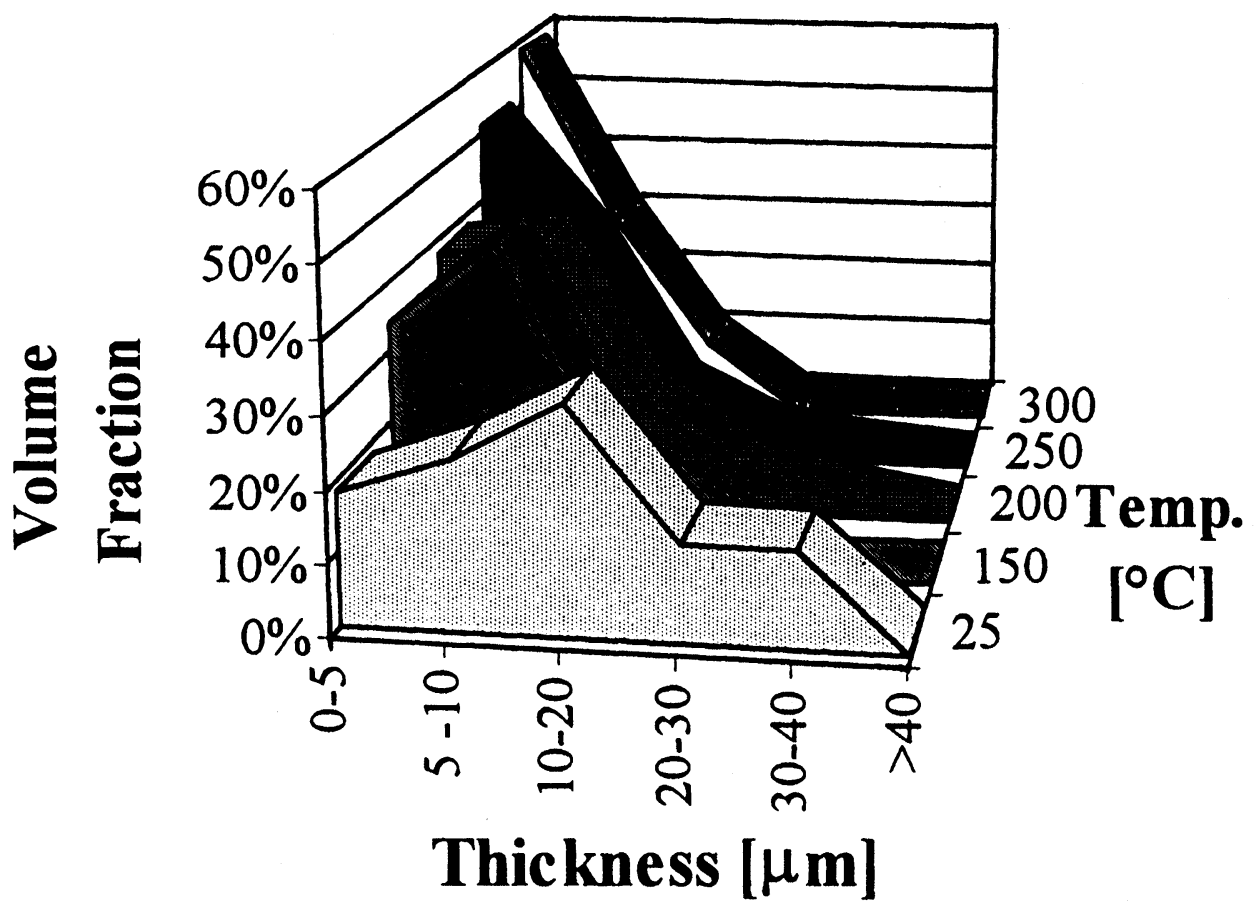

Figure 6 Thickness distribution of orientation layers after $70 \%$ compression at various deformation temperatures.

Texture and the effect of orientation on deformation banding tendency

Inverse pole figures plotting the orientation of individual layers are shown in Figure 7. Deformation textures in samples compressed below $300^{\circ} \mathrm{C}$ are similar with a major $\{110\}$ component and a minor $\{100\}$ component. It should be pointed out that the $\{100\}$ components are in fact weaker than they appear in the inverse pole figures. This is because although there is a considerable number of near $\{100\}$ oriented layers, almost all of them are thin $(<5 \mu \mathrm{m})$ and have small volume fractions. The texture in samples compressed at $300^{\circ} \mathrm{C}$ is dominated by a single $\{100\}$ component (figure 7e). Comparing to samples deformed at lower temperatures, layers with near $\{100\}$ orientations were much less frequently found in samples compressed at $300^{\circ} \mathrm{C}$.

The effect of orientation on the deformation banding tendency can also be observed from Figure $7 \mathrm{f}$, which shows the orientations of all layers (at all deformation temperatures) thicker than $30 \mu \mathrm{m}$. Apparently, most of the thick layers have near $\{110\}$ orientation and none has near $\{110\}$ orientations. In agreement with Barrett and Levenson's (1940) observation in aluminium single crystals, the present results suggest that grains with $\{110\}$ orientations have the lowest banding tendency. On the other hand, all the near $\{100\}$ oriented regions are thin and exist as deformation bands. 

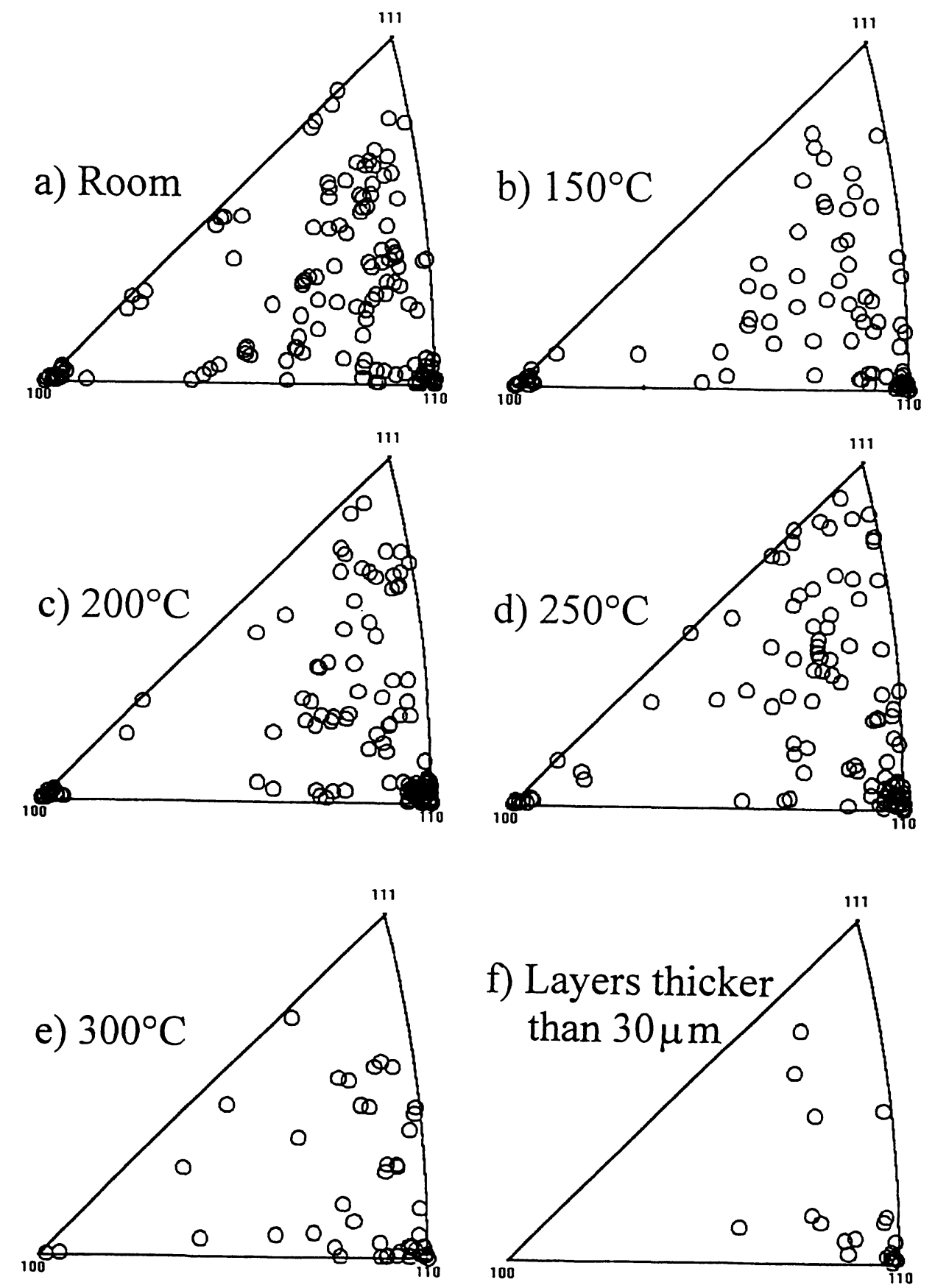

Figure 7 Inverse pole figures showing orientations of individual layers after $70 \%$ compression at a) room temperature, b) 150 , c) 200 , d) 250 , e) $300^{\circ} \mathrm{C}$ and f) orientations layers (of all deformation temperatures studied) thicker than $30 \mu \mathrm{m}$. 
The role of deformation banding in deformation at intermediate or high temperatures

It is interesting to note that while the $\{110\}$ component is predicted by the Full Constraints Taylor model for the formation of texture; neither the Full Constraints Taylor model nor the Relaxed Constraints Taylor model can predict the $\{100\}$ component (Lee et al., to be published). This is consistent with the hypothesis that the $\{100\}$ component is formed as a result of deformation banding and therefore cannot be predicted by models based on homogeneous deformation. As boundaries of deformation bands are favourable sites for recrystallisation, it is suspected that the decay of the $\{100\}$ component at $300^{\circ} \mathrm{C}$ is due to its destruction by preferential occurrence of dynamic recrystallisation.

The average layer thickness measurements in the present work clearly suggest that the deformation banding tendency increases as deformation temperature increases. On the other hand, there are reports that deformation bands are not formed during high temperature deformation (Hu, 1961; Maurice and Driver, 1993). However, it should be noted that the deformation temperatures used in these works (about $0.7 \mathrm{~T}_{\mathrm{m}}$ ) are much higher that used here (about $0.4 \mathrm{~T}_{\mathrm{m}}$ ). At such high temperatures, additional slip systems may be activated and the work hardening behaviour will be different from that of the present studies. Further work on the banding tendency at higher deformation temperatures $\left(>300^{\circ} \mathrm{C}\right)$ and other strain rates is being carried out to elucidate the deformation behaviour of copper at high temperatures (Lee et al., to be published).

\section{CONCLUSIONS}

It is observed that the deformation banding tendency in compressed copper increases as the deformation temperature increases from room temperature to $300^{\circ} \mathrm{C}$. An orientation effect was also found such that grains with near $\{110\}$ orientations have the lowest banding tendency. Textures of the compressed samples consist mainly of a $\{110\}$ fibre together with a minor $\{100\}$ component. Almost all the $\{100\}$ oriented regions exist as thin deformation bands. The $\{100\}$ component decays when the deformation temperature reaches $300^{\circ} \mathrm{C}$ where dynamic recrystallisation was observed. It is suspected that preferential occurrence of recrystallisation at heavily banded regions might be a possible cause for the decay of the $\{100\}$ component.

\section{Acknowledgement}

The authors would like to acknowledge Mr T.F. Hung and Mr K.C. Lee for help in preparation of photographic materials. The present work was supported by a City University research grant (No 700366).

\section{References}

Akef, A. and Driver, J. H. (1991). Mater. Sci. Engg., A132, 245.

Bay, B., Hansen, N., Hughes, D. A. and Kuhlmann-Wilsdorf, D. (1992). Acta Metall. Mater., $20,205$.

Bay, B., Hansen, N. and Kuhlmann-Wilsdorf, D. (1989). Mater. Sci. Engg., A113, Barrett, C. S. (1939). Trans. AIME, 135, 296.

Barrett, C. S. (1943). The Structure of Metals, 1st Ed. McGraw-Hill, p. 381-419.

Barrett, C. S. and Levenson, L. H. (1940). Trans. AIME, 137, 112.

Chin, G. Y. (1969). Texture in Research and Practice, Springer Verlag, Berlin, p. 263. 385.

Goodman, S. R. and Hu, H. (1964). Trans. AIME, 230, 1413.

Hu, H. (1961). Trans. AIME, 221, 130.

Hu, H. (1963). Recover and Recrystallisation of Metals, Interscience, New York, p. 311. 
Hu, H. (1969). Textures in Research and Practice, Springer, Berlin, p. 200.

Hu, H. and Cline, R. S. (1961). J. Appl. Phys., 32, 760.

Hu, H., Cline, R. S. and Goodman, S. R. (1961). J. Appl. Phys., 32, 1392.

Hu, H. and Cline, R. S. (1965). Trans. AIME 233, 310.

$\mathrm{Hu}, \mathrm{H}$. and Goodman, S. R. (1963). Trans. AIME, 227, 627.

Hughes, D. A. and Hansen, N. (1991) Mater. Sci. Technol., 7, 544.

Köhlhoff, G. D., Sun, X. and Lücke, K, (1988). Proc. $8^{\text {th }}$ Int. Conf. on Textures of Materials, p. 183.

Lee, C. S. and Duggan, B. J. (1993). Acta Metall. Mater., 41, 2691.

Lee, C. S., Duggan, B. J. and Smallman, R. E. (1993a). Acta Metall. Mater., 41, 2265.

Lee, C. S., Duggan, B. J. and Smallman, R. E. (1993b). Phil. Mag. Let., 68, 185.

Lee, C. S., Lee, K. C., Lai, J. K. L. and Smallman, R. E., to be publish.

Mott, N. F. (1951). Proc. Phys. Soc. B, 64, 729.

Rosen, G. L., Juul Jensen, D., Hughes, D. A. and Hansen, N. (1995). Acta Metall. Mater., 43, 2563.

Maurice, Cl. and Driver, J. H. (1993). Acta Metall. Mater., 41, 1653. 